\title{
A CONCISE PROOF OF THE "GEOMETRIC" CONSTRUCTION OF INERTIAL MANIFOLDS
}

\author{
James C. Robinson \\ Department of Applied Mathematics and Theoretical Physics, \\ Silver Street, Cambridge, CB3 9EW, \\ England.
}

\begin{abstract}
By employing the standard Strong Squeezing Property, the "geometric" existence proof of Constantin et al. [1] is considerably simplified, and the parallels between this and other methods thereby emphasised.
\end{abstract}




\section{Introduction}

Inertial manifolds (finite-dimensional, positively invariant, exponentially attracting Lipschitz manifolds) were introduced in the mid-80s [2] in an attempt to understand the finite-dimensional behaviour observed in many dissipative partial differential equations. Since then there have been several different methods of proof (well summarised in ref. [3]), based either on analytical methods (the fixed point approach of ref. [4] and many others, or the method of "elliptic regularisation" of ref. [5]) or more geometric ones: the "graph transform" method of ref. [6] or the "Cauchy" method of ref. [1]. In many of these arguments some form of "cone condition" [6,7] plays a part. Of central importance in the graph transform method, the cone condition can also be related to the fixed point method [7] in a way that closely links the two arguments.

In [7] it was also shown that a strengthened version of the cone condition, the strong squeezing property [8], is sufficient to ensure existence of an inertial manifold. This was done using a mixture of geometric and analytical ideas. However, combining the strong squeezing property with the Cauchy method significantly shortens both methods of proof.

\section{Brief formulation of the problem and definitions}

Study of inertial manifolds has been centred on equations of the form

$$
d u / d t+A u+f(u)=0,
$$

where $u \in H$, a Hilbert space, $A$ is a positive self-adjoint linear operator, and $f$ is a Lipschitz map from $D\left(A^{\alpha}\right)$ into $D\left(A^{\beta}\right)(0 \leq \alpha-\beta \leq 1 / 2)$. By using the existence in $D\left(A^{\alpha}\right)$ of a bounded absorbing set, it is possible to truncate $f[10]$ so that it is zero outside some sphere $\Omega_{\rho}$ in $D\left(A^{\alpha}\right)$,

$$
\begin{gathered}
\operatorname{supp}(f) \subset \Omega_{\rho} \equiv\left\{u:\left|A^{\alpha} u\right| \leq \rho\right\}, \\
\sup _{u \in \Omega_{\rho}}\left|A^{\beta} f(u)\right| \leq C_{0} \forall u \in D\left(A^{\alpha}\right), \text { and } \\
\left|A^{\beta}\left(f\left(u_{1}\right)-f\left(u_{2}\right)\right)\right| \leq C_{1}\left|A^{\alpha}\left(u_{1}-u_{2}\right)\right| \forall u_{1}, u_{2} \in D\left(A^{\alpha}\right),
\end{gathered}
$$

where $|\cdot|$ is the norm on $H$. Such an equation generates a strongly continuous semigroup $S(t)$ on $D\left(A^{\alpha}\right)$, so that for initial conditions $u_{0} \in D\left(A^{\alpha}\right)$, $u\left(t ; u_{0}\right)=S(t) u_{0}$ (see ref. [9]). It is therefore natural to measure distances with respect to the norm in $D\left(A^{\alpha}\right)$. 
Since its inverse is compact, $A$ has an orthonormal basis of eigenvectors $w_{j}$, with corresponding eigenvalues which will be ordered to be nondecreasing,

$$
A w_{j}=\lambda_{j} w_{j} \quad \lambda_{j+1} \geq \lambda_{j} .
$$

One can then define the finite-dimensional projection operators $P_{n}$ by

$$
P_{n} u=\sum_{j=1}^{n}\left(u, w_{j}\right) w_{j}
$$

where $(\cdot, \cdot)$ is the inner product on $H$. Together with each $P_{n}$ there is the orthogonal (if $\lambda_{n+1} \neq \lambda_{n}$ ) projection $Q_{n} u=\left(I-P_{n}\right) u$. All inertial manifolds so far obtained have been given as the graph of a Lipschitz function $\phi$ : $P_{n} H \rightarrow Q_{n} H \cap D\left(A^{\alpha}\right)$, i.e.

$$
\begin{gathered}
\mathcal{M}=\mathcal{G}[\phi] \equiv\left\{p+\phi(p): p \in P_{n} H\right\}, \text { with } \\
\left|A^{\alpha}\left(\phi\left(p_{1}\right)-\phi\left(p_{2}\right)\right)\right| \leq l\left|A^{\alpha}\left(p_{1}-p_{2}\right)\right|
\end{gathered}
$$

The proof presented below will use the strong squeezing property, which combines the existence of an invariant cone with the exponential decay of the difference of trajectories outside the cone.

Definition. Let $u_{1}(t)$ and $u_{2}(t)$ be two solutions of (1). The strong squeezing property holds if for some $N$ and $l$

$$
\left|A^{\alpha} Q_{N}\left(u_{1}(0)-u_{2}(0)\right)\right| \leq l\left|A^{\alpha} P_{N}\left(u_{1}(0)-u_{2}(0)\right)\right|
$$

implies that

$$
\left|A^{\alpha} Q_{N}\left(u_{1}(t)-u_{2}(t)\right)\right| \leq l\left|A^{\alpha} P_{N}\left(u_{1}(t)-u_{2}(t)\right)\right|
$$

for all $t \geq 0$, and furthermore that if

$$
\left|A^{\alpha} Q_{N}\left(u_{1}\left(t_{0}\right)-u_{2}\left(t_{0}\right)\right)\right| \geq l\left|A^{\alpha} P_{N}\left(u_{1}\left(t_{0}\right)-u_{2}\left(t_{0}\right)\right)\right|
$$

then

$$
\left|A^{\alpha} Q_{N}\left(u_{1}(t)-u_{2}(t)\right)\right| \leq\left|A^{\alpha} Q_{N}\left(u_{1}(0)-u_{2}(0)\right)\right| e^{-k t}
$$

for $0 \leq t \leq t_{0}$ and some $k>0$. 
A sufficient condition for the strong squeezing property to hold is that $[7,9]$

$$
\lambda_{N+1}-\lambda_{N}>2 C_{1}\left(\lambda_{N+1}^{\alpha-\beta}+\lambda_{N}^{\alpha-\beta}\right),
$$

known as the spectral gap condition.

\section{The existence theorem}

The approach adopted combines the cone condition and the "Cauchy method" of ref. [1].

Theorem. Suppose that there exists a global attractor $\mathcal{A}$ with Hausdorff dimension $d_{H}(\mathcal{A})$, and that the strong squeezing property holds for some $N>d_{H}(\mathcal{A})+1$. Then the set $\Sigma$, given by

$$
\Sigma \equiv \overline{\bigcup_{t \geq 0} S(t) \Gamma},
$$

where $\Gamma \equiv \partial P_{N} \Omega_{\rho}$, is an inertial manifold given as the graph of a Lipschitz function. The manifold $\mathcal{M}$, defined by

$$
\mathcal{M}=\Sigma \cup\left\{u: u \in P_{N} H,\left|A^{\alpha} u\right| \geq \rho\right\},
$$

is also an inertial manifold and is asymptotically complete [11], i.e. for any trajectory $u(t)$ there exists a point $u_{0} \in \mathcal{M}$ s.t.

$$
\left|A^{\alpha}\left(S(t) u_{0}-u(t)\right)\right| \leq C(u(0)) e^{-k t} .
$$

The following method is partially suggested by a comment in ref. [12], which obtains existence by a different method related to the graph transform. Indeed, it is clear that once an inertial manifold has been constructed (3) will give the manifold. However, in the argument below (3) is used to prove the existence of such a manifold directly.

Proof. The first step is to show that $\Sigma$ is the graph of a Lipschitz function $\phi$ over $P_{N} \Omega_{\rho}$. Since $f=0$ for $\left|A^{\alpha} u\right|>\rho$, clearly

$$
\bigcup_{0 \leq t \leq t_{0}} S(t) \Gamma \subset S\left(t_{0}\right)\left\{u: u \in P_{N} H, \rho \leq\left|A^{\alpha} u\right| \leq \rho e^{\lambda_{N} t_{0}}\right\} .
$$

Thus the cone invariance part of the strong squeezing property implies that for any two points $u_{i}$ in $\cup_{0 \leq t \leq t_{0}} S(t) \Gamma$,

$$
\left|A^{\alpha} Q\left(u_{1}-u_{2}\right)\right| \leq l\left|A^{\alpha} P\left(u_{1}-u_{2}\right)\right|,
$$


since by (4) the two initial points satisfy (2). Thus the function $\phi$ defined over $P_{n} \Sigma$ is Lipschitz over its domain of definition. All that remains to show that $P_{n} \Sigma \supset P_{n} \Omega_{\rho}$. Since the semigroup is continuous, $P_{n} \Sigma$ is connected. Furthermore, the dimension of the attractor is less than $N-1$, so $\mathcal{A}$ cannot divide the manifold (cf. ref. [12]). Thus $P_{n} \Sigma \supset P_{n} \Omega_{\rho} . \Sigma$ is positively invariant by construction, as is $\mathcal{M}$. It remains only to prove the asymptotic completeness property of $\mathcal{M}$.

Define $V(u)$ (cf. refs. [7] and [11]) by

$$
V(u)=\left\{v \in \mathcal{M}:\left|A^{\alpha} Q w\right| \geq l\left|A^{\alpha} P w\right|, w=v-u\right\}
$$

the intersection of $\mathcal{M}$ with the complement of the invariant cone from the strong squeezing property. It is easy to show that $V(u)$ is compact, since it is closed and bounded: for any $v=\pi+\phi(\pi)$ in $V(u), l\left|A^{\alpha} \pi\right| \leq(1+l)\left|A^{\alpha} u\right|+\|\phi\|$ (a bound on $\|\phi\|=\sup _{p \in P H}\left|A^{\alpha} \phi\right|$ can be easily obtained using the variation of constants formula). Now consider a sequence of times $t_{n} \rightarrow \infty$ and points $v\left(t_{n}\right) \in V_{t_{n}}$ (i.e. $\left.V\left(u\left(t_{n}\right)\right)\right)$. By the negative invariance of $V_{t}$, $u(n)=S\left(-t_{n}\right) v\left(t_{n}\right) \in V_{0}$, and by compactness of $V_{0}$ there exists a limit point of the $u(n), u_{0} \in V_{0}$, such that $S(t) u_{0} \in V_{t}$ for all $t \geq 0$. Thus, by the strong squeezing property,

$$
\begin{aligned}
\left|A^{\alpha}\left(u(t)-S(t) u_{0}\right)\right| & \leq\left(1+l^{-1}\right)\left|A^{\alpha} Q\left(u(t)-S(t) u_{0}\right)\right| \\
& \leq\left(1+l^{-1}\right)\left|A^{\alpha} Q\left(u(0)-u_{0}\right)\right| e^{-k t} \\
& \leq\left(1+l^{-1}\right)\left(\mid A^{\alpha} Q(u(0))+\|\phi\|\right) e^{-k t}
\end{aligned}
$$

which is asymptotic completeness, and clearly implies exponential convergence to $\mathcal{M}$.

\section{Conclusion}

It has been shown that when the strong squeezing property holds (and provided $N$ is large enough) an inertial manifold is given by

$$
\overline{\bigcup_{t \geq 0} S(t) \Gamma}, \quad \text { where } \quad \Gamma \equiv \partial P_{N} \Omega_{\rho} .
$$

Furthermore, the eventual behaviour of any trajectory $u(t)$ is given by some trajectory $v(t)$ lying on the manifold, so that

$$
\left|A^{\alpha}(u(t)-v(t))\right| \sim e^{-k t}
$$


ensuring that the behaviour on the manifold does indeed capture all the asymptotic dynamics of the system.

The importance of the strong squeezing property is highlighted by its use as a basis for the "Cauchy" method, while the proof itself becomes much more transparent. Furthermore, this approach is extremely useful when trying to use eq. (5) to numerically approximate the inertial manifold, as it makes such a construction easier to apply to discrete versions of eq. (1).

\section{Acknowledgement}

This work was been supported by Trinity College, Cambridge. I am very grateful to them for all their generosity.

\section{References}

[1] P. Constantin, C. Foias, B. Nicolaenko and R. Temam, Integral manifolds and inertial manifolds for dissipative partial differential equations (Springer, Berlin, 1988).

[2] C. Foias, G.R. Sell and R. Temam, C. R. Acad. Sci. I 301 (1985) 139.

[3] R. Temam, Math. Intell. 12 (1990) 68.

[4] S.-N. Chow, K. Lu and G.R. Sell, J. Math. Anal. Appl. 169 (1992) 283.

[5] A. Debussche, Diff. Integral Eq. 3 (1990) 467.

[6] J. Mallet-Paret and G.R. Sell, J. Amer. Math. Soc. 1 (1988) 805.

[7] J.C. Robinson, Dyn. Sys. Appl. 2 (1993) 311.

[8] C. Foias, B. Nicolaenko, G.R. Sell and R. Temam, J. Math. Pures Appl. 67 (1988) 197.

[9] D. Henry, Geometric theory of semilinear parabolic equations (Springer, New York, 1981).

[10] R. Temam, Infinite dimensional dynamical systems in mechanics and physics (Springer, Berlin, 1988).

[11] C. Foias, G.R. Sell and E.S. Titi, J. Dyn. Diff. Eq. 1 (1989) 199.

[12] P. Constantin, C. Foias, B. Nicolaenko and R. Temam, J. Dyn. Diff. Eq. (1989) 45. 